\title{
Competing Nonlinear Delocalization of Light for Laser Inscription Inside Silicon with a $2-\mu \mathrm{m}$ Picosecond Laser
}

\author{
M. Chambonneau, ${ }^{1}$ L. Lavoute, ${ }^{2}$ D. Gaponov, ${ }^{2}$ V.Y. Fedorov, ${ }^{3,4}$ A. Hideur, ${ }^{5}$ S. Février, ${ }^{6}$ \\ S. Tzortzakis, ${ }^{3,7,8}$ O. Utéza, ${ }^{1}$ and D. Grojo ${ }^{1, *}$ \\ ${ }^{1}$ Aix-Marseille Univ., CNRS, LP3 UMR 7341, 13009 Marseille, France \\ ${ }^{2}$ Novae, ZI du Moulin Cheyroux, 87700 Aixe-sur-Vienne, France \\ ${ }^{3}$ Science Program, Texas A\&M University at Qatar, P.O. Box 23874, Doha, Qatar \\ ${ }^{4}$ P. N. Lebedev Physical Institute of the Russian Academy of Sciences, 53 Leninskiy Prospekt, 119991 Moscow, \\ Russia \\ ${ }^{5}$ CNRS, INSA, Univ. Rouen, CORIA UMR 6614, 76801 Rouen, France \\ ${ }^{6}$ Univ. Limoges, CNRS, XLIM UMR 7252, 87000 Limoges, France \\ ${ }^{7}$ Institute of Electronic Structure and Laser (IESL), Foundation for Research and Technology-Hellas (FORTH), \\ P.O. Box 1527, GR-71110 Heraklion, Greece \\ ${ }^{8}$ Materials Science and Technology Department, University of Crete, 71003 Heraklion, Greece
}

(Received 23 January 2019; revised manuscript received 20 May 2019; published 5 August 2019)

\begin{abstract}
The metrology of laser-induced damage usually finds a single transition from $0 \%$ to $100 \%$ damage probability when progressively increasing the laser energy in experiments. We observe that picosecond pulses at $2-\mu \mathrm{m}$ wavelength focused inside silicon provide a response that strongly deviates from this. Supported by nonlinear propagation simulations and energy flow analyses, we reveal an increased light delocalization for near critical power conditions. This leads to a nonmonotonic evolution of the peak delivered fluence as a function of the incoming pulse of the energy, a situation more complex than the clamping of the intensity until now observed in ultrafast regimes. Compared to femtosecond lasers, our measurements show that picosecond sources lead to reduced thresholds for three-dimensional (3D) writing inside silicon that is highly desirable. However, strong interplays between nonlinear effects persist and should not be ignored for the performance of future technological developments. We illustrate this aspect by carefully retrieving from the study the conditions for a demonstration of 3D data inscription inside a silicon wafer.
\end{abstract}

DOI: 10.1103/PhysRevApplied.12.024009

\section{INTRODUCTION}

Different emerging short-pulse fiber lasers emitting in the short-wave infrared (SWIR) region of the spectrum $(1.1-2.5 \mu \mathrm{m})$ represent attractive tools to extend the threedimensional (3D) laser writing technologies developed in transparent dielectrics [1] to narrow gap materials as important as silicon ( $\mathrm{Si})[2-4]$. Nevertheless, the first femtosecond laser experiments in this prospect reveal that the intrinsic properties of narrow gap materials prevent any permanent material change in the bulk $[5,6]$ unless nonconventional focusing conditions are used [7]. This

\footnotetext{
*david.grojo@univ-amu.fr
}

Published by the American Physical Society under the terms of the Creative Commons Attribution 4.0 International license. Further distribution of this work must maintain attribution to the author(s) and the published article's title, journal citation, and DOI. is caused by a strong clamping of the intensity due to nonlinear effects developing in the prefocal region. These contributing effects include Kerr-induced phase distortions, beam depletion by the highly efficient multiphoton absorption inherent to narrow gap materials, and increased plasma effects (screening and defocusing) due to the long wavelengths that are inevitably used [8-10].

Capitalizing on the advent of erbium-doped laser technologies, the investigations have concentrated on telecommunication wavelengths $(1.3$ and $1.5 \mu \mathrm{m})$ so far. In particular, we have recently investigated in-volume structuring of Si using pulses at $1.55 \mu \mathrm{m}$ with durations varying from 0.8 to $10 \mathrm{ps}$. We found that permanent modification in the bulk was achievable, but multipulse processing with our longest pulse duration of $10 \mathrm{ps}$ was a requirement [11]. In a previous work, extreme focusing was identified as a spatial optimization to reduce the peak power and prefocal nonlinear interactions for femtosecond pulses [7]. One conclusion with the study in the picosecond regime 
was that it leads to a similar optimization in the temporal domain for bulk writing. However, it was hard to find conditions for $100 \%$ damage probability and reproducible morphologies. This raises additional questions on the specificity of the picosecond regime and its potential applications.

In this paper, we concentrate on this aspect by applying a standard methodology for laser-induced damage measurements and comparisons with nonlinear propagation simulations. While we still investigate the Si response, we introduce a longer wavelength that hold promise to process materials with even narrower band gaps. Surprisingly, we find a bulk damage probability, which does not monotonically increase with the incoming pulse energy. This is taken as clear evidence of the persistence of competing nonlinear effects affecting the delivered laser energy density at the focus. It explains the difficulties in the previous attempts that aimed at controlled internal laser structuring of $\mathrm{Si}$ in the picosecond regime [11]. From this study, we retrieve conditions for repeatable writing and the successful inscription of data at separated surface and volume planes of a Si wafer.

\section{EXPERIMENTAL SETUP}

In the experiments, we use a recently developed $5-\mu \mathrm{J}$ ultrafast thulium-doped fiber laser operating at $1970 \mathrm{~nm}$. Compact monolithic all-fiber systems are usually limited to sub- $\mu \mathrm{J}$ energy levels $[12,13]$. To circumvent this limitation, we apply the chirped pulse amplification technique to the seed pulses generated in a dissipative soliton oscillator [14]. This allows all-fiber integration, high seed average power, an adjustable spectrum to fit a dispersive components transmission band, and a prestretched pulse. The amplification stage features $25-\mu \mathrm{m}$ core diameter active fibers instead of rod-type photonic crystal fibers. In order to avoid nonlinear effects in the fiber at the $\mu \mathrm{J}$ level, the seed pulse is stretched up to 400 ps by means of a chirped fiber Bragg grating stretcher. The linear amplification regime is obtained up to an average output power of $500 \mathrm{~mW}$ at the repetition rate of $100 \mathrm{kHz}$ used in these experiments. As shown in Fig. 1, the amplified pulses are externally compressed using a chirped volume Bragg grating $(\mathrm{CVBG})$ down to 2 ps as measured by autocorrelation.

For the damage tests, linearly polarized pulses are tightly focused at different depths in an intrinsic Si wafer [(100)-oriented, double-side polished] using an aspherical lens of numerical aperture NA $=0.85$ (Thorlabs, C037TME-D) mounted on a motorized stage (along the optical $z$ axis). Figure 1 also shows that $x-y$ positioning is performed by the motorized motion of the Si wafer. The theoretical spot diameter is approximately equal to $1.5 \mu \mathrm{m}$ (FWHM) in air as calculated for a lens filling ratio of 0.65 [15]. The expected modifications are near or below

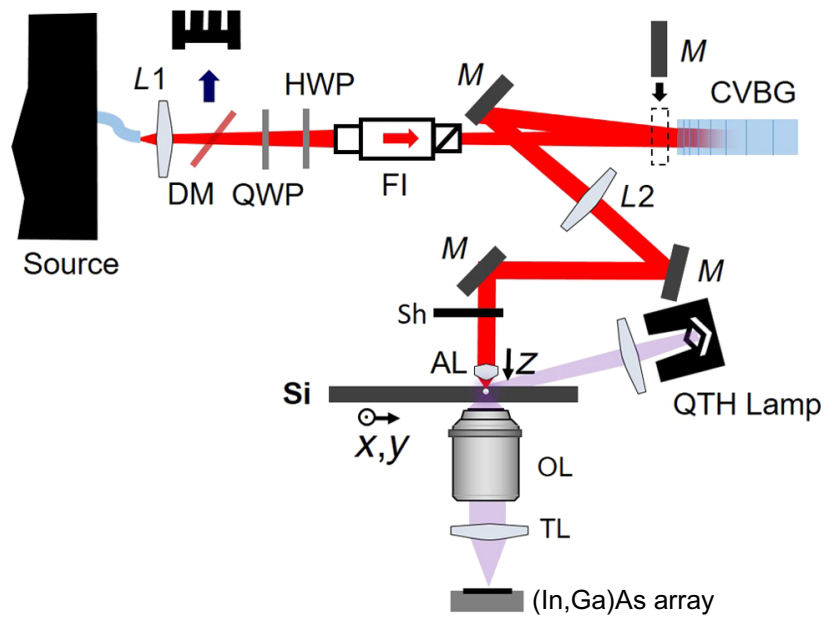

FIG. 1. Experimental arrangement for the damage tests with a $2-\mu \mathrm{m}$ laser at different pulse durations. $\mathrm{DM}$ is a dichroic mirror rejecting the pump. FI is a Faraday isolator transmitting linearly polarized pulses. QWP is a quarter-wave plate to create a nearly linear polarization after exiting the fiber. HWP is a half-wave plate on a motorized stage added to control the FI transmission and the laser pulse energy used in the experiments. CVBG is a chirped volume Bragg grating to compress the pulse down to 2 ps and can be bypassed with an inserting mirror (M). The residual divergence of the beam after L1 is exploited to collimate the beam with L2 at the position where it reaches an appropriate diameter to fill AL, the aspheric focusing lens. Sh is a mechanical shutter. For high-sensitivity modification detection, an infrared dark-field microscope is implemented. OL,TL, and QTH are, respectively, an objective, a tube lens, and a Quartz-Tungsten Halogen lamp for imaging with an (In, Ga)As camera.

the resolution limit of any infrared imaging system. Thus, we decide to employ dark-field microscopy for scattering detection of any produced damage in the bulk. In practice, the samples are illuminated at a grazing angle with a whitelight lamp and an (In, Ga)As camera is installed for in situ observation with $10 \times$ magnification (see Fig. 1).

\section{RESULTS AND DISCUSSIONS}

At first, we test several NAs, exposure times, and depths of focusing. For the measurements, we choose to apply Son-1 sequences of $10^{4}$ pulses focused with $\mathrm{NA}=0.85$ at a depth of $150 \mu \mathrm{m}$ inside the Si wafer. Linear propagation calculations end with strong spherical aberrations at this depth. This leads to a fluence reduction by more than $60 \%$ in comparison to vacuum, where the fluence would exceed $50 \mathrm{~J} \mathrm{~cm}^{-2}$ at our maximum laser energy [15]. However, we observe that these conditions ensure confined modification into the bulk over a significant energy range. For comparison, focusing at $\mathrm{NA}=0.56$ does not allow us to achieve any bulk modification with 2-ps pulses. While we could have employed higher repetition rates, we choose to perform the exposures at $100 \mathrm{kHz}$ for safely avoiding any thermal accumulation on a shot-to-shot basis $[11,16]$. 


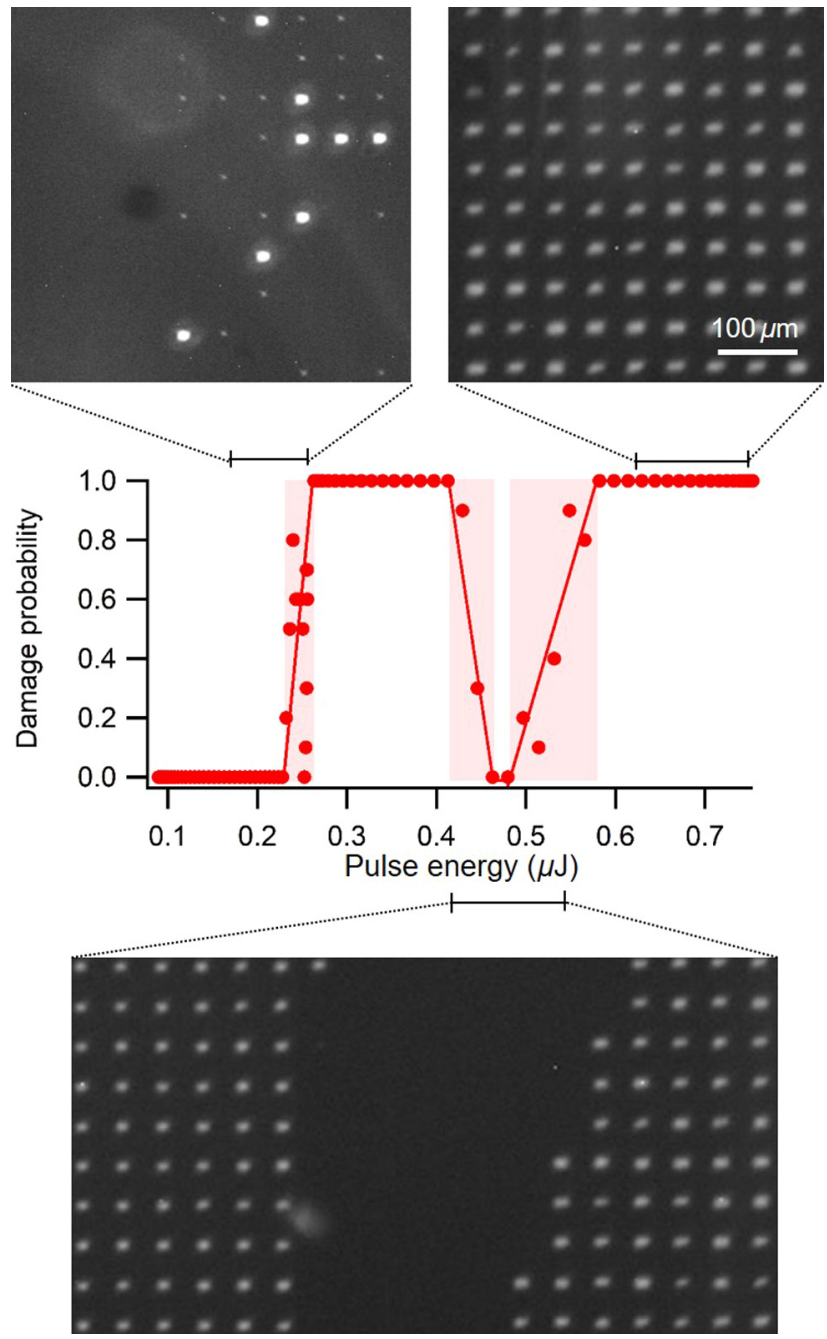

FIG. 2. Energy dependence of the damage probability with 2-ps pulses focused $(\mathrm{NA}=0.85)$ at $150 \mu \mathrm{m}$ below the surface of silicon. The sample is irradiated with gradually increasing energies on fresh sites separated by $50 \mu \mathrm{m}$. All measurements result from statistics over 10 identical irradiations corresponding to lines observed by dark-field IR microscopy as shown by the inserted images. The spatial scale applies to all inserted images. Several transitions between 0 and 100\% damage probability (color regions) evidence a nonmonotonic change of the delivered fluence due to the nonlinearities.

For statistical analyses, the irradiations are performed by automated procedures systematically repeated 10 times so that the processed region takes the form of 10 identical lines of impacts separated by $50 \mu \mathrm{m}$. Figure 2 shows the measured damage probability as a function of energy at the shortest pulse duration of 2 ps. The inserted images reveal sections of the 10 lines irradiated with increasing laser energies (from left to right). We first note a transition from 0 to $100 \%$ damage probability for a pulse energy approximately equal to $250 \mathrm{~nJ}$, thus defining the damage threshold. By looking at the top-left image, we observe that for near-threshold conditions, modifications (when occurring) stochastically take either the form of a highly localized damage site or one that has grown and is scattering light more efficiently. This stochastic behavior may originate from (i) near-threshold energy instabilities as well as (ii) a random distribution of precursor defects [17]. However, as soon as this transition region is exceeded, a highly repeatable modification is found, as is seen in the left part of the bottom image (Fig. 2). The most striking feature occurs at higher energy (around $450 \mathrm{~nJ}$ ) where the damage probability drops down to zero indicating a reduction of the delivered fluence below the damage threshold. It is also interesting to note that by increasing the energy, it remains possible to overcome this reduction to recover $100 \%$ damage probability with apparently less localized modifications as shown with the top-right image where larger scatterers are observed.

We focus our attention on this very unusual nonmonotonic damage response by repeating the experiment in several regions of the sample under various reconfigurations. We also characterize the space-time characteristics of the beam for all laser conditions. While the width of the damage transitions could slightly change, the systematic observation of an absence of damage between 0.45 and $0.55 \mu \mathrm{J}$ confirms that the observed feature is not related to precursor defects in the sample or an uncontrolled change of the beam characteristics during the experiments.

There are always limits to the energy density that can be delivered in a material with intense laser pulses due to intensity clamping. For surface experiments, it is the development of screening effects (e.g., plasma mirror), which limits the energy deposition. For bulk experiments, the limit is usually reached at reduced power because the nonlinear interactions develop in the material in the prefocal region $[7,18]$. However, since the observation in Fig. 2 exceeds a saturation or a clamping effect, it can only be explained by a delocalization of the laser energy under certain conditions. To examine this problem theoretically, we apply our recently demonstrated transformation optics approach for a simplified treatment of highly nonparaxial nonlinear problems [10]. Our model accounts for the same set of physical mechanisms as in our previous work in the femtosecond regime, including nonlinear Kerr refraction, nonlinear photoionization, and the associated plasma effects. The quantitative agreement between the measured and calculated modification thresholds is not as good as for our previous experiments with aberrationfree femtosecond pulses [7]. This is possibly due to some aberrations because of the use of a focusing lens far from its design wavelength. Nevertheless, in the tested energy range $(0.01-0.75 \mu \mathrm{J}$ corresponding to ratios to the critical power $P / P c$ between about 0.2 and 15), we systematically find a minimum in the peak delivered fluence. This directly correlates with the damagefree window observed in Fig. 2 for above threshold conditions. One way to reveal the delocalization is to rely on an energy flow 


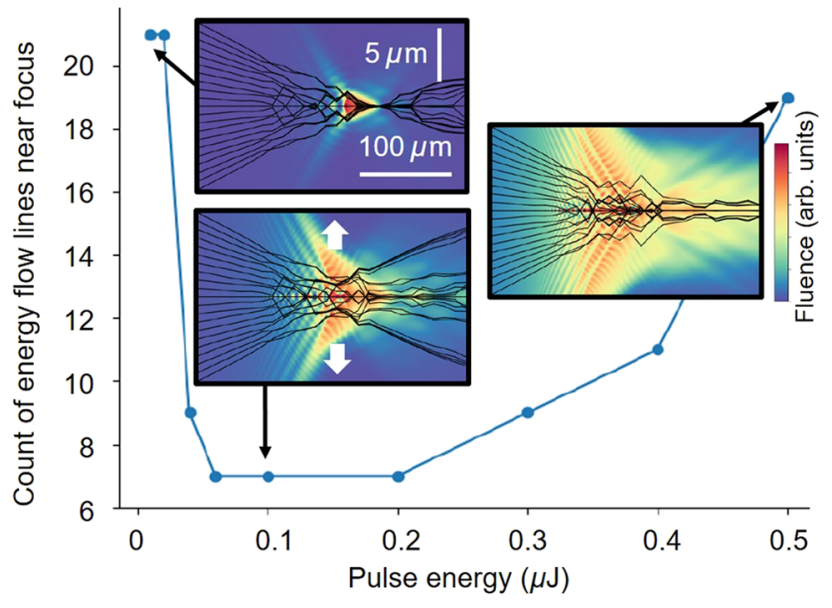

FIG. 3. Energy flow analysis by nonlinear propagation simulations of focused picosecond pulses. Nonlinear propagation simulations for 2-ps pulse at $2-\mu \mathrm{m}$ wavelength are performed to map the energy flow inside the material near the focal region (NA) at a depth of $150 \mu \mathrm{m}$. The insets show the fluence distribution and energy flow lines obtained by retrieving the wave vectors from the wavefront of the pulse at each propagation distance for incoming energies of $0.01,0.1$, and $0.5 \mu \mathrm{J}$. The graph gives the number of energy flow lines reaching a zone of $1 \mu \mathrm{m}$ at the vicinity of the focus as a function of the input pulse energy. A minimum corresponding to a lower angular component contributing to the focus is found when varying the pulse energy. The structured fluence distribution originates from diffraction for a beam overfilling the entrance pupil of the lens. The Supplemental Material [19] provides a more complete energy flow analysis under various assumptions on the spatial characteristics of the beam.

analysis. Figure 3 shows the results for a beam overfilling the entrance pupil of a $0.85 \mathrm{NA}$ lens (diffracted). At each propagation distance, we calculate the fluence and the phase of the picosecond laser pulse. By mapping in space the wave vectors perpendicular to the wavefront, the energy flow can be displayed. While the complete analysis is provided in the Supplementary Material [19], we illustrate the procedure with the images embedded in Fig. 3, where the corresponding energy flow lines are superimposed on the calculated fluence distributions for incoming pulse energies of $0.01,0.1$, and $0.5 \mu \mathrm{J}$. Finally, to quantify the delocalization of light as a function of energy, we calculate how many energy flow lines reach a zone of $1-\mu \mathrm{m}$ width around the focus. This leads to the graph of Fig. 3, where one identifies the range where the energy flow into the focal volume is minimal. We also notice with the simulations that relocalization of light preferentially occurs in the secondary maxima of the diffracted beam (see, for instance, Fig. 4(a) of Supplemental Material [19]). This encourages us to look at the dependence of the beam profile as shown in the Supplemental Material [19]. Smooth Gaussian focusing leads to a less pronounced effect, confirming that hot spots in the prefocal beam structure likely help
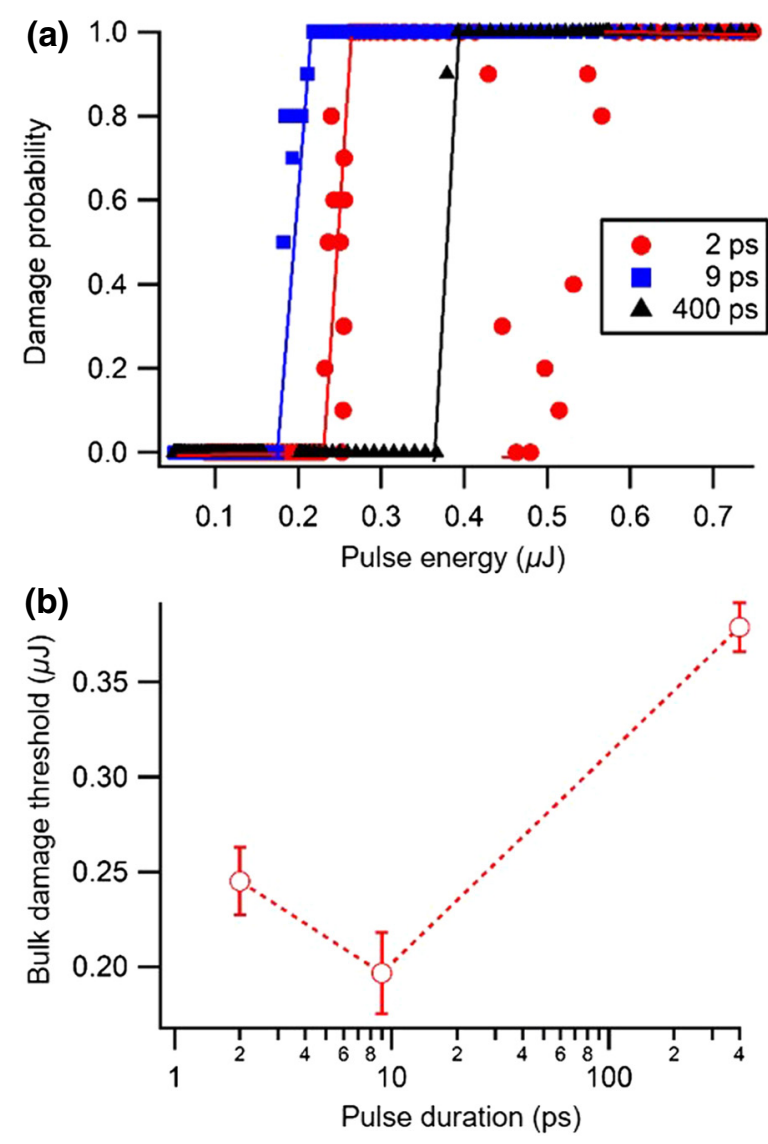

FIG. 4. Influence of the pulse duration on the bulk damage response with pulses focused $(\mathrm{NA}=0.85)$ at $150 \mu \mathrm{m}$ below the surface of silicon. All measurements result from statistics over 10 identical irradiations to report the damage probability as a function of the incoming pulse energy (b). Only the shortest pulses ( 2 ps) exhibit multiple transitions. For comparison, the damage threshold as a function of the tested pulse durations is shown (b). The vertical bars stand for the energy range between the highest tested energy without any modification and the lowest energy exhibiting 100\% modification. The lowest threshold for modification is found for the intermediate pulse duration of $9 \mathrm{ps}$.

energy relocalization, but are not a prerequisite (see Supplemental Material for the profile-dependence study [19]). The obtained similar effect for a smooth Gaussian beam can be taken as indirect evidence of the important role of Kerr focusing as it is the only nonlinear effect accounted for in our model that can reconcentrate the energy flux.

To confirm that the energy delocalization and unconventional damage response is a specificity of the interplay between nonlinear effects inside $\mathrm{Si}$ in the picosecond regime, we complement the measurements by experiments (i) with longer pulses to gradually reduce the peak power in $\mathrm{Si}$ and (ii) at the surface to totally suppress the prefocal interaction (maximum intensity of approximately $10^{9} \mathrm{~W} \mathrm{~cm}^{-2}$, which is too low for interaction in air). Two ways have been employed for changing the pulse duration. 
First, we add dispersion by electrically adjusting the temperature gradients on the stretcher grating element so that the pulse exiting the CVBG shown in Fig. 1 can exhibit durations ranging from 2 to $10 \mathrm{ps}$. Second, to access much longer pulses, we use uncompressed pulses of 400-ps duration by simply bypassing the CVBG as shown in Fig. 1. The results of the damage measurements for three different pulse durations (2, 9, and $400 \mathrm{ps})$ are displayed in Fig. 4. A first observation is the classical monotonic behavior for the damage probability with 9-ps and 400-ps pulses in the bulk as well as on the entrance surface for all pulse durations. This demonstrates that the laser energy delocalization and relocalization originate from a nonlinear competition in the bulk of $\mathrm{Si}$, which is strong enough at a peak power level only accessible with our shortest pulses in the tested energy range. While we cannot definitively exclude a drop of the damage probability (above threshold) for pulse energies out of this range when pulses longer than 2 ps are studied, it is important to highlight that the fluence drop found in the simulations is relatively modest $(6 \%$ for best cases as shown in Fig. 5 of Supplemental Material [19]). A consequence is that the drop can translate into a nonmonotonic damage response only for pulses delivering saturated fluences, which are near-threshold conditions. As mentioned above, $2 \mathrm{ps}$ and 0.85 are the shortest pulse duration and smallest NA for which bulk modifications are achievable with our source, respectively. Accordingly, we assume it is unlikely that conditions that significantly deviate from these near-threshold conditions will indirectly reveal any delocalization and/or relocalization of the laser energy by the material damage response (unless much stronger similar effects are accessible at high energies). To confirm this vision, we have returned to the $1550-\mathrm{nm}$ wavelength that was leading to hardly controllable laser writing. While we found a different pulse-duration threshold for writing in the conditions of our new experiments, we qualitatively observe the same nonmonotonic damage response for nearthreshold conditions. This was likely causing the lack of repeatability in our previous experiments in the picosecond regime [11].

Figure 4 also shows a decrease of the damage threshold value when the duration increases from 2 ps to 9 ps. This confirms our previous observations of increasing difficulties in achieving bulk modifications when decreasing the pulse duration [11] with a material response that culminates with the incapacity to exceed the modification threshold in the femtosecond laser experiments performed at various wavelengths due to even more pronounced nonlinear effects affecting the propagation $[6,8,20]$.

This dependence on the pulse duration is opposite to the one observed for surface damage measurements, as we find a monotonic increase of damage threshold with respect to the pulse duration (from $1.6 \mathrm{~J} \mathrm{~cm}^{-2}$ at $2 \mathrm{ps}$ and $1.9 \mathrm{~J} \mathrm{~cm}^{-2}$ at 9 ps to $4.6 \mathrm{~J} \mathrm{~cm}^{-2}$ at $400 \mathrm{ps)}$ in such a damage test configuration where the nonlinear propagation aspects are


FIG. 5. Bulk-surface two-plane data inscription by laser machining. The sample is observed by (a) SEM and (b) IR dark field microscopy $(\mathrm{NA}=0.45$ ) focused at the surface and (c) at the bulk-written plane below the surface. The bulk text is first inscribed with consecutive irradiations of sites with tightly focused pulses $\left(2 \times 10^{5}\right.$ pulses at $\left.100 \mathrm{kHz}\right)$ at a fixed pulse energy above the bulk damage threshold $(320 \mathrm{~nJ})$. Machining of the surface text is performed in a second step using the same procedure with pulses at reduced energy $(50 \mathrm{~nJ})$. The two planes are clearly distinguished with IR microscopy and there is nothing related to the bulk inscription revealed by SEM imaging of the surface. The same scale bar applies to all images.

completely suppressed. Interestingly, we note in Fig. 4(b) that 400-ps pulses for which we can expect that the nonlinear propagation aspects vanish in the bulk due to the low beam power $(<<\mathrm{kW})$ also lead to an increased damage threshold. In both experiments (surface and volume), long pulses give nonlinear ionization only a seeding role in the energy deposition process. When the pulse duration largely exceeds the thermalization time (through electronphonon coupling) on the order of a picosecond [1], thermal 
runaway assists energy deposition by band-gap closure. These interactions are usually governed by thermal diffusion, implying that the measured thresholds scale as the square root of the pulse duration [21]. On the side of modeling, a consequence is the temperature dependence of silicon nonlinearities that must be accounted for. This is the reason the propagation simulations performed for the shortest pulse duration of 2 ps are not repeated for longer pulses.

Finally, in order to show the potential of $2-\mu \mathrm{m}$ picosecond sources for laser writing inside narrow gap materials, we perform data inscription in two distinct planes. The pulse energy is first set at $320 \mathrm{~nJ}$ for reliable digital writing at a depth of $150 \mu \mathrm{m}$ below the surface of a Si wafer. To achieve well-localized modifications, we choose this energy, which is above the first damage threshold and below the first drop of the damage probability observed with the 2-ps pulses (see Fig. 2). We apply sequences on individual pixels separated by $20 \mu \mathrm{m}$ to write one of the affiliations of the authors of this paper. Data inscription of a second affiliation is performed in a second step using the same procedure at a reduced pulse energy $(50 \mathrm{~nJ})$ to avoid overlap of the affected zones due to the low threshold for surface ablation. The observations of the processed sample are shown in Fig. 5. The data in the two planes are clearly distinguished with our microscopy system [Figs. 5(b) and 5(c)], except that we have increased the magnification to $20 \times$ for a reduced depth of field. When focusing the imaging system on one of the processed planes, we can barely detect the information in the other plane. A scanning electron microscopy image is shown in Fig. 5(a). This shows that surface structuring is achieved by laser ablation and there is absolutely nothing on the surface that is related to the inscription in the bulk. In addition to the technological considerations, another interest of this demonstration is that it unambiguously shows that we did not create any confusion between bulk and surface modifications in this report.

\section{CONCLUSION}

In conclusion, intensity clamping is a well-studied phenomenon inside wide band-gap systems, where monotonic damage probability behavior is observed. The case of narrow band-gap material reveals an additional complexity in the energy flux. Our results show the existence of strongly competing nonlinear effects modulating the fluence delivery in the picosecond regime. We reveal and quantify this modulation by looking at the bulk-Si modification response with a $2-\mu \mathrm{m}$ picosecond laser. However, we conclude that delocalization and relocalization of intense light is a general feature that should not be ignored in hardto-3D machine materials because processing is inevitably achieved in near-threshold conditions. With this knowledge, we successfully identify a window for controllable writing inside $\mathrm{Si}$ in the picosecond regime. Moreover, we anticipate a direct benefit of the thulium-doped fiber laser technologies used in these experiments with the possibility for bulk processing of even narrower band-gap materials (e.g., germanium). This represents an additional challenge in this rapidly growing field of research after the recent demonstrations of the first in-chip functional microsystems fabricated inside Si by laser writing [2-4,22].

\section{ACKNOWLEDGMENTS}

This research has been performed in the frame of the International Associated Laboratory "MINOS" and has received funding from the European Research Council (ERC) under the European Union's Horizon 2020 research and innovation program (Grant Agreement No. 724480). We also acknowledge partial funding support by the National Priorities Research Program Grant No. NPRP9383-1-083, from the Qatar National Research Fund (member of The Qatar Foundation), and the EU H2020 Laserlab Europe (Grant No. EC-GA 654148).

[1] R. R. Gattass and E. Mazur, Femtosecond laser micromachining in transparent materials, Nat. Photonics 2, 219 (2008).

[2] M. Chambonneau, Q. Li, M. Chanal, N. Sanner, and D. Grojo, Writing waveguides inside monolithic crystalline silicon with nanosecond laser pulses, Opt. Lett. 41, 4875 (2016).

[3] I. Pavlov, O. Tokel, S. Pavlova, V. Kadan, G. Makey, A. Turnali, Ö Yavuz, and FÖ Ilday, Femtosecond laser written waveguides deep inside silicon, Opt. Lett. 42, 3028 (2017).

[4] O. Tokel, A. Turnall, G. Makey, P. Elahi, T. Çolakoğlu, E. Ergeçen, Ö Yavuz, R. Hübner, M. Zolfaghari Borra, I. Pavlov, A. Bek, R. Turan, D. K. Kesim, S. Tozburun, S. Ilday, and FÖ Ilday, In-chip microstructures and photonic devices fabricated by nonlinear laser lithography deep inside silicon, Nat. Photonics 11, 639 (2017).

[5] V. V. Kononenko, V. V. Konov, and E. M. Dianov, Delocalization of femtosecond radiation in silicon, Opt. Lett. 37, 3369 (2012).

[6] A. Mouskeftaras, A. V. Rode, R. Clady, M. Sentis, O. Utéza, and D. Grojo, Self-limited underdense microplasmas in bulk silicon induced by ultrashort laser pulses, Appl. Phys. Lett. 105, 191103 (2014).

[7] M. Chanal, V. Y. Fedorov, M. Chambonneau, R. Clady, S. Tzortzakis, and D. Grojo, Crossing the threshold of ultrafast laser writing in bulk silicon, Nat. Commun. 8, 773 (2017).

[8] D. Grojo, S. Leyder, P. Delaporte, W. Marine, M. Sentis, and O. Utéza, Long-wavelength multiphoton ionization inside band-gap solids, Phys. Rev. B 88, 195135 (2013).

[9] E. V. Zavedeev, V. V. Kononenko, and V. I. Konov, Delocalization of femtosecond laser radiation in crystalline $\mathrm{Si}$ in the mid-IR range, Laser Phys. 26, 016101 (2016).

[10] V. Y. Fedorov, M. Chanal, D. Grojo, and S. Tzortzakis, Accessing Extreme Spatiotemporal Localization of HighPower Laser Radiation through Transformation Optics and 
Scalar Wave Equations, Phys. Rev. Lett. 117, 043902 (2016).

[11] H. Kämmer, G. Matthäus, S. Nolte, M. Chanal, O. Utéza, and D. Grojo, In-volume structuring of silicon using picosecond laser pulses, Appl. Phys. A 124, 302 (2018).

[12] P. Wan, L.-M. Yang, and J. Liu, High pulse energy $2 \mu \mathrm{m}$ femtosecond fiber laser, Opt. Express 21, 1798 (2013).

[13] F. Tan, H. Shi, R. Sun, P. Wang, and P. Wang, $1 \mu \mathrm{J}$, sub-300 fs pulse generation from a compact thulium-doped chirped pulse amplifier seeded by Raman shifted erbium-doped fiber laser, Opt. Express 24, 22461 (2016).

[14] D. Gaponov, L. Lavoute, S. Février, A. Hideur, and N. Ducros, $2 \mu \mathrm{m}$ all-fiber dissipative soliton master oscillator power amplifier, Proc. SPIE 9728, 972834 (2016).

[15] M. J. Nasse and J. C. Woehl, Realistic modeling of the illumination point spread function in confocal scanning optical microscopy, J. Opt. Soc. Am. A 27, 295 (2010).

[16] D. G. Papazoglou and S. Tzortzakis, Physical mechanisms of fused silica restructuring and densification after femtosecond laser excitation, Opt. Mater. Express 1, 625 (2011).
[17] N. Bloembergen, Laser-induced electric breakdown in solids, IEEE J. Quantum Electron 10, 375 (1974).

[18] A. Mouskeftaras, M. Chanal, M. Chambonneau, R. Clady, O. Utéza, and D. Grojo, Direct measurement of ambipolar diffusion in bulk silicon by ultrafast infrared imaging of laser-induced microplasmas, Appl. Phys. Lett. 108, 041107 (2016).

[19] See Supplemental Material at http://link.aps.org/supple mental/10.1103/PhysRevApplied.12.024009 for the propagation simulations.

[20] A. H. Nejadmalayeri, P. R. Herman, J. Burghoff, M. Will, S. Nolte, and A. Tünnermann, Inscription of optical waveguides in crystalline silicon by mid-infrared femtosecond laser pulses, Opt. Lett. 30, 964 (2005).

[21] B. Stuart, M. Feit, S. Herman, A. Rubenchik, B. Shore, and M. Perry, Nanosecond-to-femtosecond laserinduced breakdown in dielectrics, Phys. Rev. B 53, 1749 (1996).

[22] G. Matthäus, H. Kämmer, K. A. Lammers, C. Vetter, W. Watanabe, and S. Nolte, Inscription of silicon waveguides using picosecond pulses, Opt. Express 26, 4875 (2018). 